Preprint typeset in JHEP style - HYPER VERSION

KIAS-P08081

\title{
Dark matter and sub-GeV hidden $U(1)$ in GMSB models
}

\author{
Eung Jin Chun*and Jong-Chul Park ${ }^{\dagger}$ \\ Korea Institute for Advanced Study, Heogiro 87, Dongdaemun-gu, Seoul 130-722, \\ Korea
}

\begin{abstract}
Motivated by the recent PAMELA and ATIC data, one is led to a scenario with heavy vector-like dark matter in association with a hidden $U(1)_{X}$ sector below GeV scale. Realizing this idea in the context of gauge mediated supersymmetry breaking (GMSB), a heavy scalar component charged under $U(1)_{X}$ is found to be a good dark matter candidate which can be searched for direct scattering mediated by the Higgs boson and/or by the hidden gauge boson. The latter turns out to put a stringent bound on the kinetic mixing parameter between $U(1)_{X}$ and $U(1)_{Y}$ : $\theta \lesssim$ $10^{-6}$. For the typical range of model parameters, we find that the decay rates of the ordinary lightest neutralino into hidden gauge boson/gaugino and photon/gravitino are comparable, and the former decay mode leaves displaced vertices of lepton pairs and missing energy with distinctive length scale larger than $20 \mathrm{~cm}$ for invariant lepton pair mass below $0.5 \mathrm{GeV}$. An unsatisfactory aspect of our model is that the Sommerfeld effect cannot raise the galactic dark matter annihilation by more than 60 times for the dark matter mass below TeV.
\end{abstract}

KEYWORDS: dark matter, cosmic rays, cosmology of theories beyond the SM.

*ejchun@kias.re.kr

$\dagger j$ jpark@kias.re.kr 


\section{Contents}

1. Introduction 1

2. Spontaneous breaking of hidden $U(1)_{X}$ by gauge mediation 3

3. Kinetic mixing between gauge bosons/gauginos of $S U(2)_{\mathrm{L}} \times U(1)_{\mathrm{Y}}$ and a hidden $U(1)_{X}$

4. Relic density, positron/electron excess and direct detection 6

5. Visible OLSP decay and Displaced vertices of lepton pairs 10

6. Conclusion 12

\section{Introduction}

It is now firmly established that $23 \%$ of the energy density of the universe consists of an unknown particle called dark matter. Discovering the nature of dark matter would be one of the most important tasks in current and future theoretical and experimental investigations. Numerous searches for galactic dark matter have been made to observe direct signals of dark matter scattering with nuclei and indirect evidences of dark matter annihilation to various Standard Model particles. Recently, a number of stimulating results toward indirect signals have been announced.

The Payload for Antimatter Matter Exploration and Light-nuclei Astrophysics (PAMELA) collaboration has reported an excess in the positron fraction, $e^{+} /\left(e^{+}+\right.$ $e^{-}$), but no excess in anti-proton fraction $\bar{p} / p$ [1]. The observed spectrum departed from the background calculation of the cosmic-ray secondary positron spectrum [2] for energies 10-100 GeV. This recent result is comparable with less certain excesses observed in HEAT [3] and AMS-01 [4]. The PPB-BETS balloon experiment and the Advanced Thin Ionization Calorimeter (ATIC) instrument have also reported an excess in the $e^{+}+e^{-}$energy spectrum of $300-800 \mathrm{GeV}$ [5, 6].

The origin of such excesses could be astronomical objects such as a pulsar which is expected to emit energetic electron-positron pairs [7, 8, 9]. On the other hand, excessive antiparticle fluxes in galactic cosmic rays have been considered as a primary way for indirect detection of dark matter. It is encouraging that galactic dark matter annihilation can easily reproduce the amplitudes and spectral shapes of the PAMELA 
and ATIC data. However, there appear two puzzling aspects. First, the dark matter annihilation cross-section is required to be enhanced by order of 100-1000 compared with what is allowed by the standard thermal relic abundance analysis. According to a recent analysis based on $\Lambda$ CDM $N$-body simulations, however, the boost factor from clumpy matter distribution can hardly larger than about 10 [10]. Second, no distinctive excess of antiprotons in the PAMELA data disfavors most conventional dark matter candidates. A new analysis of the $\bar{p} / p$ ratio compared to the PAMELA data puts stringent limits on possible enhancements of the $\bar{p} / p$ flux from dark matter annihilation [1]]. Many ideas and models of dark matter annihilation or decay have already been suggested for the explanation of the recent observations [12, 13, 14, 15, 16, 17, 18, 19, 20, 21, 22, 23, 24, 25, 26, 27, 28, 29, 30, 31, 32, 33, 34, 35, 36, 37]. However, it appears to be a hard task to understand the nature of enhanced hadrophobic annihilation in a consistent framework with thermal dark matter.

In this paper, we elaborate the idea of dark matter charged under an extra $U(1)_{X}$ gauge group suggested in Refs. [20, 38, 39]. The presence of $U(1)_{X}$ broken at the sub$\mathrm{GeV}$ scale is motivated to explain the two intriguing features of the PAMELA data. First, the DM annihilation to extra light gauge bosons can be enhanced significantly by non-perturbative Sommerfeld effect [40, 41, 42, 43, 44]. Second, the hadrophobic nature of the $U(1)_{X}$ gauge boson decay can be understood by kinematics when its mass is below the GeV scale 20, 24]. We will consider the extra $U(1)_{X}$ gauge boson hidden from the Standard Model sector except for a small kinetic mixing between $U(1)_{X}$ and $U(1)_{Y}$, through which the $U(1)_{X}$ gauge bosons can decay mostly into lepton-antileptons. It was shown long ago that kinetic mixing can exist between two $U(1)$ gauge bosons without violating the gauge-invariance and renormalizability [45]. The mixing between an unbroken extra $U(1)$ and $U(1)_{e m}$ has been used to explain other anomalous astronomical observation such as galactic $511 \mathrm{keV} \gamma$-rays [46].

Breaking $U(1)_{X}$ below the GeV scale can arise naturally by radiative mechanism, in particular, in the context of gauge mediated supersymmetry breaking (GMSB) [39]. We will first present a concrete way of realizing such a scheme, which predicts a $U(1)_{X}$ charged scalar field as a dark matter candidate. The dark matter mass in the range of $600-1000 \mathrm{GeV}$ is preferred for the simultaneous explanation of the PAMELA and ATIC data. Unfortunately, the Sommerfeld enhancement factor of our scenario is found to be about 40-60 for this mass range. The assumption of some clumpy distribution of dark matter would be a reasonable additional source for further increase of the boost factor. Our dark matter candidate can yield observable signals for direct detection through elastic scatterings mediated by the hidden gauge boson $X$ or Higgs bosons. In the first case, we draw a severe constraint on the kinetic mixing parameter: $\theta \lesssim 10^{-6}$ for $m_{X} \sim 0.4 \mathrm{GeV}$. One of the interesting consequences of our scenario is that the ordinary lightest supersymmetric particle (OLSP), typically neutralino, decays instantaneously to the $X$ boson and its superpartner $\tilde{X}$ through 
one-loop diagram with heavy dark matter superfields in the loop. Then, the produced $X$ boson subsequently decays to two leptons with a decay length typically larger than $10 \mathrm{~cm}$, which may be observed at the LHC.

\section{Spontaneous breaking of hidden $U(1)_{X}$ by gauge mediation}

Let us start with working in gauge mediated supersymmetry breaking (GMSB) models [47] as a promising way of realizing the sub-GeV $U(1)_{X}$ sector. In such a scheme, the origin of a heavy mass of dark matter from the hidden $U(1)_{X}$ sector and the $\mu$ term can be related in the context of non-minimal supersymmetric standard model [39]:

$$
W=\frac{\lambda_{S}}{3} S^{3}+\lambda_{q} S q^{\prime} q^{\prime c}+\lambda_{H} S H_{1} H_{2}+\lambda_{\Psi} S \Psi \Psi^{c},
$$

where $\left(\Psi, \Psi^{c}\right)$ is the Dirac pair carrying $U(1)_{X}$ charge $(+1,-1)$. Note that the second term with extra quark pairs is introduced to generate sufficiently large negative masssquared for $S$ and thus a large vacuum expectation value $v_{S}$, which will lead to proper electroweak symmetry breaking [48]. A similar mechanism will be used to break $U(1)_{X}$ at the sub-GeV scale in the below. Having generated $v_{S}$, one obtains $\mu$ and $B$ terms for the Higgs fields: $\mu=\lambda_{H} v_{S}$ and $B=\lambda_{S} v_{S}$. Similarly, we also obtain the supersymmetric mass of hidden Dirac fermions $m_{\psi} \equiv \lambda_{\Psi} v_{S}$ and the corresponding (Dirac) soft bilinear term:

$$
V_{\text {soft }}=B m_{\psi} \tilde{\psi} \tilde{\psi}^{c}+\text { h.c. . }
$$

Because of this mixing mass term, two hidden scalars $\tilde{\psi}$ and $\tilde{\psi}^{c}$ have mass splitting and the mass eigenstates denoted by $\tilde{\psi}_{1,2}$ have the masses $m_{\tilde{\psi}_{1,2}}$ where $m_{\tilde{\psi}_{1,2}}^{2}=$ $m_{\psi}^{2} \mp B m_{\psi}$ taking $B$ is real positive. Therefore, the lighter scalar $\tilde{\psi}_{1}$, being lighter than the Dirac fermion $\left(\psi, \psi^{c}\right)$, will be our dark matter particle.

Let us turn to supersymmetry breaking fed into the hidden $U(1)_{X}$ sector. Note that the Dirac superfield $\left(\Psi, \Psi^{c}\right)$ plays a role of 'messenger field' for the hidden sector supersymmetry breaking whose size is controlled by the $B$ parameter. That is, the supersymmetry breaking masses of the hidden gaugino $\tilde{X}$ and the scalar component $\phi_{x}$ of a hidden superfield $\Phi_{x}$, carrying $U(1)_{X}$ charge $x$, are given by

$$
\begin{aligned}
m_{\tilde{X}} & =\frac{\alpha_{X}}{4 \pi} B \\
m_{\phi_{x}}^{2} & =2 x^{2} m_{\tilde{X}}^{2}
\end{aligned}
$$

which are defined at the 'messenger scale' $m_{\psi}$. Here $\alpha_{X}=g_{X}^{2} / 4 \pi$ is the $U(1)_{X}$ gauge fine structure constant. The reference value for the hidden gaugino mass is $m_{\tilde{X}} \approx 0.3 \mathrm{GeV}$ for $\alpha_{X}=\alpha$ and $B=500 \mathrm{GeV}$. Consider now the following hidden sector superpotential:

$$
W=\lambda_{1} \Phi_{0} \Phi_{z} \Phi_{-z}+\lambda_{p} \Phi_{0} \Phi_{p z} \Phi_{-p z}+\frac{\lambda_{0}}{3} \Phi_{0}^{3},
$$


where the subscripts denote the $U(1)_{X}$ charges. Denoting $m_{x}$ as the soft mass of the scalar component $\phi_{x}$, we get $m_{p z}^{2}=p^{2} m_{z}^{2}$ and $m_{0}^{2}=0$ at the messenger scale $m_{\psi}$. Then, one can generate a large negative mass-squared for $\phi_{0}$ down around the scale $m_{p z}$ assuming $m_{p z}^{2} \gg m_{z}^{2}(p>1)$ and the Yukawa coupling $\lambda_{p}$ of order one:

$$
m_{0}^{2} \approx-n \frac{p^{2} \lambda_{p}^{2}}{4 \pi^{2}} m_{z}^{2} \ln \frac{m_{\psi}}{p m_{z}},
$$

where $n$ is the number of the $\Phi_{ \pm p z}$ pairs. This will induce a sizable mixing vacuum expectation value $v_{0}$ of $\phi_{0}$ determined to be $v_{0}^{2}=-m_{0}^{2} / 2 \lambda_{0}^{2}$. Then the scalar potential for $\phi_{ \pm z}$ is given by

$$
V=\lambda_{1}^{2}\left|\phi_{z} \phi_{-z}\right|^{2}+\left[\lambda_{1} \lambda_{0} v_{0}^{2} \phi_{z} \phi_{-z}+\text { c.c. }\right]+\left(\lambda_{1}^{2} v_{0}^{2}+m_{z}^{2}\right)\left[\left|\phi_{z}\right|^{2}+\left|\phi_{-z}\right|^{2}\right] .
$$

If the mixing mass term of $\phi_{z} \phi_{-z}$ is larger than the diagonal mass $m_{z}^{2}+\lambda_{1}^{2} v_{0}^{2}$, there appears a direction of negative mass-squared and thus $U(1)_{X}$ symmetry breaking occurs. For the minimization of the above potential, we examine the D-flat direction $\phi_{-z}=\phi_{z}$ which has the mass eigenvalues $m_{ \pm}^{2}=2\left(m_{z}^{2}+\lambda_{1}^{2} v_{0}^{2} \pm \lambda_{1} \lambda_{0} v_{0}^{2}\right)$. To get $m_{-}^{2}<0$ and thus $v_{z}^{2} \equiv\left\langle\phi_{z}\right\rangle^{2}=-m_{0}^{2} / \lambda_{1}^{2}$, one needs to have

$$
-m_{0}^{2}>8 m_{z}^{2}
$$

assuming $\lambda_{0}=2 \lambda_{1}<\lambda_{p} \sim 1$. This condition can be generically met by adjusting the parameters $n, p$ in Eq. (2.5). Given $v_{z}$, the $X$ gauge boson gets the mass

$$
m_{X}=2 g_{X} z v_{z} .
$$

The $X$ boson mass is therefore settled down around the hidden gaugino mass scale $m_{\tilde{X}}$. However, their specific values will depend on the choice of the parameters $n, p$ and the couplings $\lambda_{0,1, p}$.

Note that the hidden $U(1)_{X}$ sector can have a communication with the Standard Model sector through the Yukawa couplings, $\lambda_{H}$ and $\lambda_{\Psi}$. In particular, our scalar dark matter $\tilde{\psi}_{1}$ can annihilate to quarks and leptons through the Higgs contact interaction:

$$
\mathcal{L}=-\frac{1}{2} \lambda_{H} \lambda_{\Psi} H_{1} H_{2} \widetilde{\psi}_{1} \widetilde{\psi}_{1}^{*}+\text { h.c. } .
$$

We want this channel to be subdominant to the dark matter annihilation into the light hidden fields as alluded in the introduction. For this purpose, we will assume

$$
\lambda_{H} \lambda_{\Psi} \ll g_{X}^{2}
$$

throughout this paper. Then, there can also be kinetic mixing between $U(1)_{X}$ and $U(1)_{Y}$ through which the $X$ gauge boson can decay to leptons as will be discussed in the next section. The interaction (2.9) will give an important contribution to dark 
matter scattering off nuclei through the Higgs exchange. In the decoupling limit of heavy neutral Higgs, the interaction between $\widetilde{\psi_{1}}$ and the light Higgs $h^{0}$ is given by

$$
\mathcal{L}=\lambda v h^{0} \widetilde{\psi}_{1} \widetilde{\psi}_{1}^{*}
$$

where $\lambda \equiv s_{\beta} c_{\beta} \lambda_{H} \lambda_{\Psi} / 2$. Here $\left\langle H_{1,2}^{0}\right\rangle=v_{1,2} / \sqrt{2}$ and $\tan \beta \equiv v_{2} / v_{1}$.

\section{Kinetic mixing between gauge bosons/gauginos of $S U(2)_{\mathrm{L}} \times$ $U(1)_{Y}$ and a hidden $U(1)_{X}$}

If $U(1)_{X}$ was unbroken, it mixes with $U(1)_{e m}$ and various interesting phenomena of milli-charged dark matter particles can occur [See [46] and references therein, and see [49] and references therein for the Stueckelberg extension with kinetic mixing]. When $U(1)_{X}$ is broken, the massive $X$ gauge boson mixes essentially with the $Z$ boson and leads to phenomenological consequences drastically different from the pervious case. Consider the gauge kinetic terms of $S U(2)_{\mathrm{L}} \times U(1)_{\mathrm{Y}} \times U(1)_{X}$ including the kinetic mixing term:

$$
\mathcal{L}_{\text {mixing }}=-\frac{\sin \theta}{2} \hat{B}^{\mu \nu} \hat{X}_{\mu \nu}
$$

The canonical form of the gauge kinetic term can be made by the following transformation:

$$
\left(\begin{array}{l}
\hat{B}_{\mu} \\
\hat{X}_{\mu}
\end{array}\right)=\left(\begin{array}{cc}
\sec \theta & 0 \\
-\tan \theta & 1
\end{array}\right)\left(\begin{array}{c}
B_{\mu}^{\prime} \\
X_{\mu}^{\prime}
\end{array}\right) .
$$

After the $S U(2)_{L} \times U(1)_{Y} \times U(1)_{X}$ breaking, the canonical gauge fields get masses and mixing. Diagonalizing the gauge boson mass matrix, one finds the relation between the original gauge eigenstates $\hat{W}^{3}, \hat{B}$, and $\hat{X}$ and mass eigenstates $Z, A$, and $\mathrm{X}$ :

$$
\left(\begin{array}{c}
\hat{W}_{\mu}^{3} \\
\hat{B}_{\mu} \\
\hat{X}_{\mu}
\end{array}\right)=\left(\begin{array}{ccc}
c_{W} & s_{W} & -\frac{c_{W} s_{W} m_{Z}^{2}}{m_{Z}^{2}-m_{X}^{2}} \theta \\
-s_{W} & c_{W} & -\frac{c_{W}^{2} m_{Z}^{2}-m_{X}^{2}}{m_{Z}^{2}-m_{X}^{2}} \theta \\
\frac{s_{W} m_{Z}^{2}}{m_{Z}^{2}-m_{X}^{2}} \theta & 0 & 1
\end{array}\right)\left(\begin{array}{c}
Z_{\mu} \\
A_{\mu} \\
X_{\mu}
\end{array}\right)+\mathcal{O}\left(\theta^{2}\right) .
$$

Let us now present some couplings relevant for our discussions in the limit of $\theta \ll 1$ and $m_{X} \ll m_{Z}$. The scalar dark matter $\widetilde{\psi}_{1}$ has the gauge interactions with $X$ and $Z$ as follows:

$$
\mathcal{L}=i g_{X}\left(\widetilde{\psi}_{1}^{*} \partial^{\mu} \widetilde{\psi}_{1}-\partial^{\mu} \widetilde{\psi}_{1}^{*} \widetilde{\psi}_{1}\right)\left(X_{\mu}+\theta s_{W} Z_{\mu}\right)+g_{X}^{2} \widetilde{\psi}_{1}^{*} \widetilde{\psi}_{1} X^{\mu} X_{\mu}
$$

The hidden gauge boson $X$ couples also to the visible sector particles, which is described by

$$
\mathcal{L}=\theta g^{\prime} X_{\mu} \bar{f} \gamma^{\mu} \Gamma_{f X} f
$$

with $\Gamma_{f X} \approx-Q_{f} c_{W}^{2}$ and $\Gamma_{\nu X}=-\frac{m_{X}^{2}}{2 m_{Z}^{2}} P_{L}$, 
where $Q_{f}$ is the electromagnetic charge of a quark or lepton and again the subleading terms of order of $m_{X}^{2} / m_{Z}^{2}$ are neglected except for neutrinos. In addition, the coupling of $X W W, g_{X W W}$, is given by $g_{X W W}=-\theta s_{W} g_{Z W W}$ where $g_{Z W W}$ is the SM coupling of $Z W W$.

The supersymmetric counterpart of Eq. (3.2) can be read straightforwardly [50], that is, the original hidden gaugino can be replaced by the relation,

$$
\hat{\tilde{X}}=\tilde{X}-\theta \tilde{B}
$$

leading to the bino interaction to the hidden sector fermion and scalar. In the right-hand side of the above equation, the gauginos can be considered as the mass eigenstates as the mixing mass $\theta m_{\tilde{X}}$ between $\tilde{X}$ and $\tilde{B}$ arising from the previous consideration can be safely neglected in our discussion. The coupling of bino to light hidden sector bosons and fermions given by

$$
\mathcal{L}=\theta g_{X} \tilde{B} \tilde{\phi} \phi^{*}+\text { h.c. }
$$

provides a decay channel of the ordinary lightest supersymmetric particle in the visible sector.

\section{Relic density, positron/electron excess and direct detection}

With the assumption of Eq. (2.10) and $\theta \ll 1$, which will be discussed shortly, we can neglect the dark matter annihilation to the Standard Model particles through Higgs and $Z, X$ gauge boson channels, namely, $\widetilde{\psi}_{1} \widetilde{\psi}_{1}^{*} \rightarrow H H, X Z$ and $Z Z$. Then, the dark matter relic density is determined by the process $\widetilde{\psi}_{1} \widetilde{\psi}_{1}^{*} \rightarrow X X$ having the cross-section:

$$
\langle\sigma v\rangle_{X X} \simeq \frac{4 \pi \alpha_{X}^{2}}{m_{\widetilde{\psi}_{1}}^{2}}
$$

The present relic density of $\widetilde{\psi}_{1}$ is then given by

$$
\begin{aligned}
\Omega_{\widetilde{\psi}_{1}} h^{2} & \simeq \frac{1.07 \times 10^{9} \mathrm{GeV}^{-1}}{M_{p l}} \frac{x_{F}}{\sqrt{g_{*}}} \frac{1}{\langle\sigma v\rangle_{X X}} \\
& \approx \frac{2.17 \times 10^{-10} \mathrm{GeV}^{-2}}{\langle\sigma v\rangle_{X X}},
\end{aligned}
$$

where $g_{*}$ counts the number of relativistic degrees of freedom and $x_{F} \equiv m / T_{F}$ [51]. The observed value of $\Omega_{\mathrm{DM}} h^{2} \simeq 0.1143$ [52] fixes the hidden gauge coupling constant in terms of the dark matter mass:

$$
\alpha_{X} \simeq 1.58 \alpha\left(\frac{m_{\widetilde{\psi}_{1}}}{\mathrm{TeV}}\right) .
$$




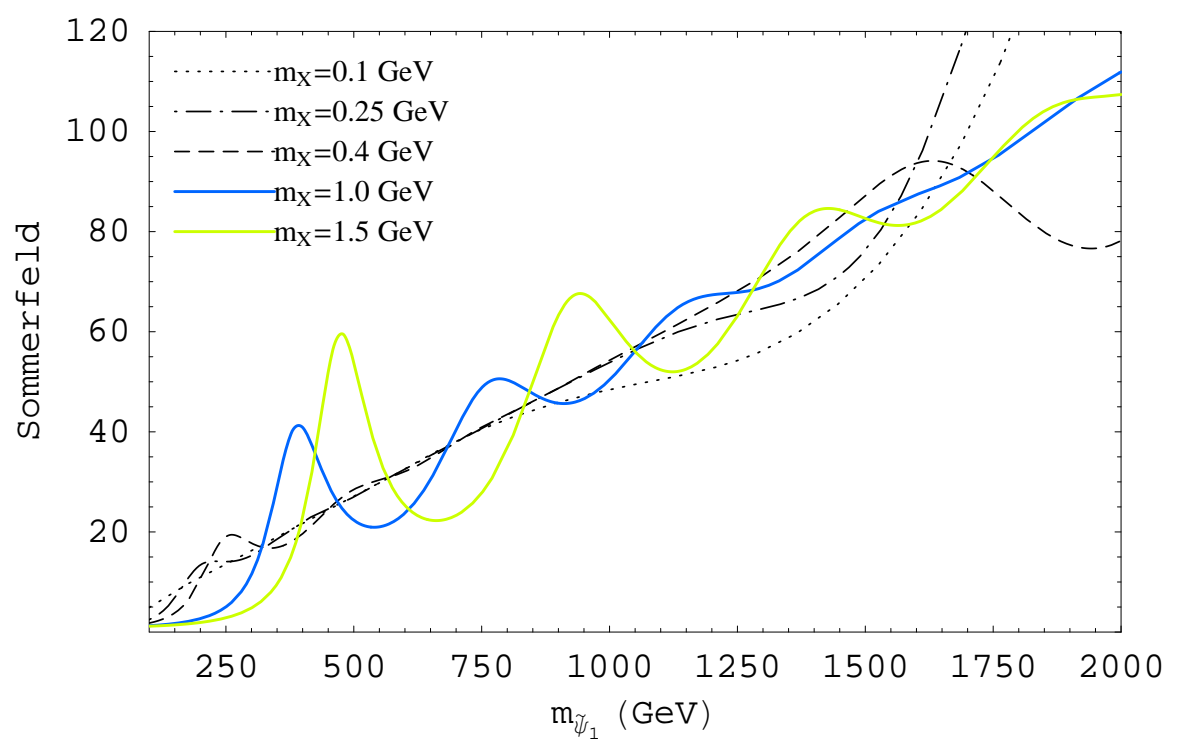

Figure 1: The Sommerfeld enhancement factor $S$ as a function of $m_{\widetilde{\psi}_{1}}$ for various $m_{X}$.

One of the troubles for the thermal dark matter explaining the recent positron/electron excesses from the galactic dark matter annihilation is the big gap between the cross section $\langle\sigma v\rangle_{\text {GAL }}$ required by the PAMELA and ATIC results and the cross section $\langle\sigma v\rangle_{\text {F.O. }}$ required by the dark matter relic density at the epoch of freeze-out. Their ratio is shown to be

$$
\frac{\langle\sigma v\rangle_{\mathrm{GAL}}}{\langle\sigma v\rangle_{\mathrm{F} . \mathrm{O} .}} \sim 10^{2-3}\left(\frac{M}{\mathrm{TeV}}\right)^{2}=B_{e} S
$$

where $B_{e}$ is the boost factor for the positron/electron and $S$ is the Sommerfeld enhancement factor 18]. In our model, the Sommerfeld enhancement arises due to the light gauge boson, and is calculated as $S=\left|\tilde{\psi}_{1}(\infty) / \tilde{\psi}_{1}(0)\right|^{2}$ after solving the following equation:

$$
-\frac{1}{m_{\tilde{\psi}_{1}}} \frac{d^{2} \tilde{\psi}_{1}(r)}{d r^{2}}-\frac{\alpha_{X}}{r} e^{-m_{X} r} \tilde{\psi}_{1}(r)=m_{\tilde{\psi}_{1}} \beta^{2} \tilde{\psi}_{1}(r)
$$

with the out-going boundary condition, $\tilde{\psi}_{1}^{\prime}(\infty) / \tilde{\psi}_{1}(\infty)=i m_{\tilde{\psi}_{1}} \beta$ where $\beta$ is the dark matter velocity 18, 20, 41, 42, 43, 44. Requiring the relic density condition (4.3), the Sommerfeld factor becomes a function of two input parameters, $m_{\widetilde{\psi}_{1}}$ and $m_{X}$, which is shown in Fig. 1. As can be seen, the Sommerfeld enhancement factor is around 50 for $m_{\widetilde{\psi}_{1}} \sim 800 \mathrm{GeV}$, and increases almost linearly as $m_{\widetilde{\psi}_{1}}$ increases except resonance effect for certain values. Our enhancement factor turns out to be insufficient to explain the PAMELA/ATIC data. This may indicate the presence of the combined effect with the boost factor $\lesssim 10$ from clumpy dark matter distribution [10]. 


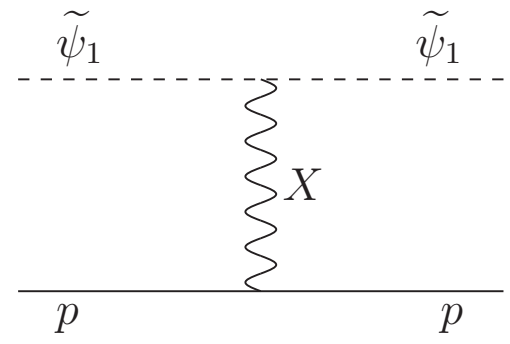

(a) vector interaction

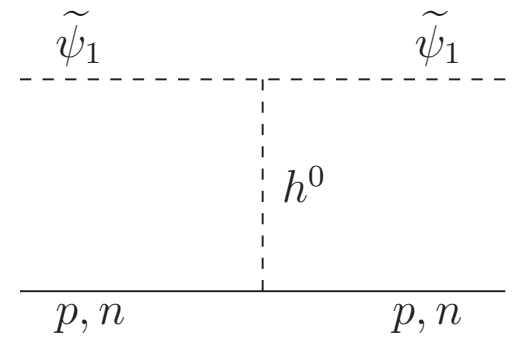

(b) scalar interaction

Figure 2: Diagrams relevant to $\widetilde{\psi}_{1}$-nucleon elastic scattering.

Given the interactions (2.11,3.4, 3.5) of the hidden matter to the visible particles, some of our parameter space is constrained by dark matter experiments for direct detection. First, the dark matter $\widetilde{\psi}_{1}$ has the effective vector interactions with quarks due to their couplings to the $X$ gauge boson:

$$
\mathcal{L}_{\text {vector }}^{q}=b_{q} i\left(\partial^{\mu} \widetilde{\psi}_{1}^{*} \widetilde{\psi}_{1}-\widetilde{\psi}_{1}^{*} \partial^{\mu} \widetilde{\psi}_{1}\right) \bar{q} \gamma^{\mu} q,
$$

where $b_{q} \equiv \theta g_{X} g^{\prime} c_{W}^{2} Q_{q} / m_{X}^{2}$. Now that the vector current is conserved, the contributions of each quark in a nucleus add coherently. In addition, sea quarks and gluons cannot contribute to the vector current. Thus, the $\widetilde{\psi}_{1}$-proton/neutron interactions can be expressed with the replaced couplings, $b_{p}=2 b_{u}+b_{d}$ and $b_{n}=b_{u}+2 b_{d}$. Note that we have $b_{n} \approx 0$ as $X$ couples to the electromagnetic charge in the leading order. As a result, the $\tilde{\psi}_{1}$-nucleus interaction is given by

$$
\mathcal{L}_{\text {vector }}^{N}=b_{N} i\left(\partial^{\mu} \widetilde{\psi}_{1}^{*} \widetilde{\psi}_{1}-\widetilde{\psi}_{1}^{*} \partial^{\mu} \widetilde{\psi}_{1}\right) \bar{N} \gamma^{\mu} N
$$

where $b_{N}=Z b_{p}+(A-Z) b_{n}=\theta g_{X} g^{\prime} c_{W}^{2} Z / m_{X}^{2}$ and the nucleus $N$ has the atomic number $Z$ and the atomic weight $A$. Therefore, the standard total cross section for the $\widetilde{\psi}_{1}$-nucleus vector interaction is given by 53

$$
\begin{aligned}
\sigma_{\text {vector }}^{\widetilde{\psi}_{1}-N} & =\frac{m_{\widetilde{\psi}_{1}}^{2} m_{N}^{2} b_{N}^{2}}{64 \pi\left(m_{\widetilde{\psi}_{1}}+m_{N}\right)^{2}} \\
& \simeq 1.25 \times 10^{4} \frac{\bar{m}_{\widetilde{\psi}_{1}}^{2} \bar{m}_{N}^{2}}{\left(\bar{m}_{\widetilde{\psi}_{1}}+\bar{m}_{N}\right)^{2}} \theta^{2} \frac{\alpha_{X}}{\alpha} \frac{Z^{2}}{\bar{m}_{X}^{4}} \mathrm{pb},
\end{aligned}
$$

where $\bar{m}_{i} \equiv m_{i} / \mathrm{GeV}$. Recall that the vector interaction leads only to spin-independent cross-section. To compare with experiments, we plot the $\widetilde{\psi}_{1}$-proton cross section for vector interactions given by

$$
\sigma_{\text {vector }}^{\widetilde{\psi}_{1}-p}=\sigma_{\text {vector }}^{\widetilde{\psi}_{1}-N} \frac{1}{Z^{2}} \frac{\mu_{p}^{2}}{\mu_{N}^{2}}
$$




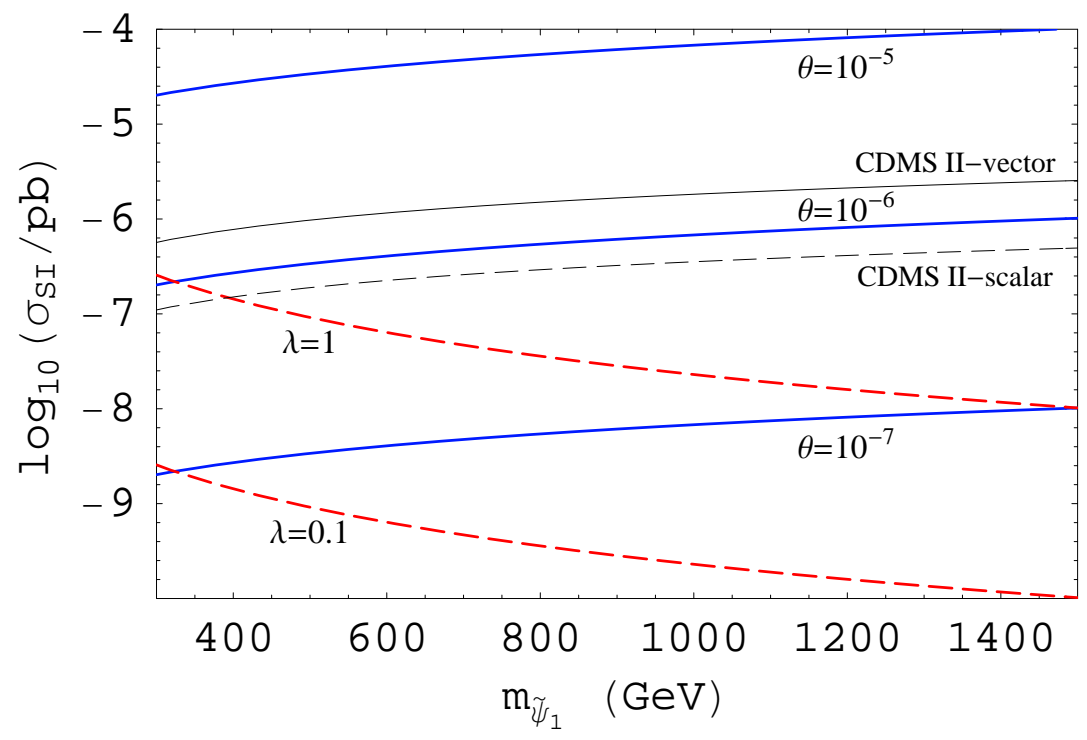

Figure 3: Exclusion plot for the spin-independent $\tilde{\psi}_{1}$-nucleon cross-section $\sigma_{\mathrm{SI}}$. The solid lines are the cross-sections via vector interactions with $m_{X}=0.4 \mathrm{GeV}$ corresponding to $\theta=10^{-5}, 10^{-6}$, and $10^{-7}$, respectively. The dashed lines are through Higgs exchange corresponding to $\lambda=1$ and 0.1 , respectively, taking $m_{h}=115 \mathrm{GeV}$. The thin solid (dashed) line shows the CDMS II limit for the vector (scalar) interaction.

where $\mu_{p, N}$ are the reduced masses for the $\widetilde{\psi}_{1}$-proton and $\widetilde{\psi}_{1}$-nucleus.

In addition to the vector interaction, the scalar dark matter $\widetilde{\psi}_{1}$ interacts with nucleons through $t$-channel Higgs exchange driven by Eq. (2.11). The $\widetilde{\psi}_{1}$-nucleon cross section for the scalar interaction is adequately estimated in [54]:

$$
\begin{aligned}
\sigma_{\text {scalar }}^{\widetilde{\psi}_{1}-n, p} & \approx\left(\frac{\lambda 0.34 \mathrm{GeV}}{m_{h}^{2} \pi}\right)^{2}\left(\frac{m_{p}}{m_{\widetilde{\psi}_{1}}+m_{p}}\right)^{2} \\
& \simeq 4.56 \times 10^{6} \frac{\lambda^{2}}{\bar{m}_{h}^{4}} \frac{\bar{m}_{p}^{2}}{\left(\bar{m}_{\widetilde{\psi}_{1}}+\bar{m}_{p}\right)^{2}} \mathrm{pb}
\end{aligned}
$$

where $\bar{m}_{i} \equiv m_{i} / \mathrm{GeV}$. If the Standard Model Higgs and hidden light higgs have the contact interaction term, $\lambda_{\phi h} \phi^{2} h^{2}$, the hidden higgs mixes with the SM Higgs when both fields have vacuum expectation values. Therefore, the t-channel exchange of the light hidden scalar through this mixing possibly dominates in some cases [55]. However, in our model, the visible and hidden sectors are separated, and so no such contact interaction exists at the tree-level. The t-channel light scalar exchange can effectively arise only from three-loop diagrams, which can be safely neglected.

The Korea Invisible Mass Search (KIMS) experiment provides the most stringent limit on the spin-dependent interaction for a pure proton case [56], and the Cryogenic Dark Matter Search (CDMS II) experiment sets the strongest limit on the 
spin-independent WIMP-nucleon interaction for a WIMP mass larger than $\sim 100$ $\mathrm{GeV}$ [57. However, the latter is only relevant in this analysis. In Fig. 3, we present the $\tilde{\psi}_{1}$-nucleon cross-sections via vector interactions (solid lines) as a function of $m_{\widetilde{\psi}_{1}}$ for typical kinetic mixing parameters, $\theta=10^{-5}, 10^{-6}$, and $10^{-7}$. The scalar interaction cross-sections (dashed lines) are also shown as a function of $m_{\widetilde{\psi}_{1}}$ for $\lambda=1$ and 0.1. The limits from CDMS II experiment is shown as thin line respectively for the vector and scalar interaction. The vector interaction puts a strong bound on the kinetic mixing parameter:

$$
\theta \leq 2 \times 10^{-6}\left(\frac{m_{X}}{0.4 \mathrm{GeV}}\right)^{2}
$$

whereas the scalar interaction puts almost no bound on $\lambda=s_{\beta} c_{\beta} \lambda_{H} \lambda_{\Psi} / 2$. We remark that in the framework of the inelastic dark matter [58] one can evade this stringent bound on the kinetic mixing parameter $\theta$ as discussed in [20], where the dark matter is part of a multiplet of a non-Abelian gauge group and small mass splittings exist between these states. In this case, the most stringent bound on kinetic mixing comes from the anomalous magnetic moment of muon: $\theta \lesssim 9 \times 10^{-3}\left(m_{X} / 0.4 \mathrm{GeV}\right)[59$.

\section{Visible OLSP decay and Displaced vertices of lepton pairs}

In our scenario, the ordinary lightest supersymmetric particle (OLSP) $\chi_{1}^{0}$, which is typically a linear combination of neutralinos including singlino $\tilde{S}$, can decay to the hidden sector particles either through the kinetic mixing (3.7) or through the singlino coupling to the dark matter superfield components $\psi, \tilde{\psi}(2.1)$. The latter leads to the OLSP decay into hidden gauge boson $X$ and gaugino $\tilde{X}$ resulting from the oneloop induced $\tilde{S}-X-\tilde{X}$ interaction as shown in Fig. 1 . The corresponding effective Lagrangian is

$$
\begin{aligned}
\mathcal{L} & =\frac{1}{2}\left(C_{1}+C_{2}\right) \overline{\widetilde{X}} \sigma^{\mu \nu} \widetilde{S} X_{\mu \nu} \\
\text { with } \quad C_{i} & =\frac{m_{\psi}}{16 \pi^{2} m_{i}^{2}} \frac{\lambda_{\Psi} g_{X}^{2}}{\sqrt{2}} J\left(x_{i}\right),
\end{aligned}
$$

where $x_{i}=m_{\psi}^{2} / m_{i}^{2}, m_{1,2}^{2} \equiv m_{\widetilde{\psi}_{1,2}}^{2}=m_{\psi}^{2} \mp B m_{\psi}$, and $J(x) \equiv \frac{1}{(1-x)^{3}}[-2+2 x-(1+$ $x) \ln x]$. In the limit of $x \rightarrow 1$, we have $J(x)=1 / 6$ and the decay rate of the singlino $\tilde{S}$ becomes

$$
\Gamma(\widetilde{S} \rightarrow X \widetilde{X})=\frac{1}{8 \pi} m_{\widetilde{S}}^{3}\left(C_{1}+C_{2}\right)^{2} \approx \frac{\lambda_{\Psi}^{2} \alpha_{X}^{2}}{2304 \pi^{3}} \frac{m_{\widetilde{S}}^{3}}{m_{\psi}^{2}},
$$

if the singlino is the OLSP. In the case of the bino OLSP, the decay rate of the bino $\tilde{B}$ to hidden higgs and higgsinos $(\phi$ and $\widetilde{\phi})$ via the kinetic mixing is given by

$$
\Gamma(\widetilde{B} \rightarrow \phi \widetilde{\phi}) \approx \frac{\alpha_{X} \theta^{2}}{2} m_{\widetilde{B}}
$$



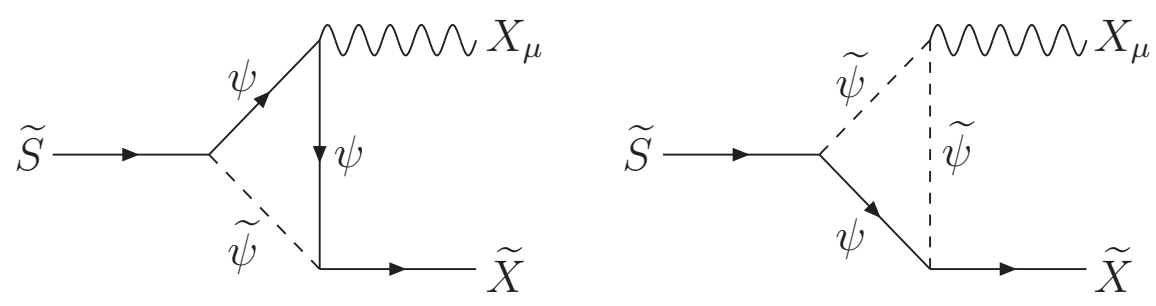

Figure 4: One-loop diagrams responsible for the decay of singlino $\widetilde{S}$ to $X \widetilde{X}$.

Considering the OLSP having the bino (singlino) component with a fraction $c_{\widetilde{B}}\left(c_{\widetilde{S}}\right)$, we get the ratio between two decay modes as

$$
\begin{aligned}
\frac{c_{\widetilde{B}}^{2} \Gamma(\widetilde{B} \rightarrow \phi \widetilde{\phi})}{c_{\widetilde{S}}^{2} \Gamma(\widetilde{S} \rightarrow X \widetilde{X})} \approx & 5 \times 10^{-4}\left(\frac{c_{\widetilde{B}}}{c_{\widetilde{S}}}\right)^{2}\left(\frac{0.1}{\lambda_{\Psi}^{2}}\right)\left(\frac{\theta}{10^{-6}}\right)^{2} \\
& \left(\frac{m_{\psi}}{700 \mathrm{GeV}}\right)^{2}\left(\frac{200 \mathrm{GeV}}{m_{\widetilde{\chi}_{1}^{0}}}\right)^{2},
\end{aligned}
$$

where we have used Eq. (4.3) and assumed $m_{\widetilde{\psi}_{1}} \approx m_{\psi}$ for simplicity. Thus, we can conclude that $\chi_{1}^{0}$ mostly decays to a hidden gauge boson and gaugino $X \tilde{X}$ in a reasonable choice of the parameter space. From the above calculations, the decay length of $\chi_{1}^{0}$ is determined to be

$$
l_{\chi_{1}^{0} \rightarrow X \widetilde{X}} \approx\left(\frac{0.1}{c_{\widetilde{S}}}\right)^{2}\left(\frac{0.1}{\lambda_{\Psi}^{2}}\right)\left(\frac{200 \mathrm{GeV}}{m_{\widetilde{\chi}_{1}^{0}}}\right)^{3} \times 10^{-3} \mathrm{~cm}
$$

ensuring that the OLSP decays well inside a detector. The decay-produced hidden gauge boson $X$ decays back to the Standard Model fermion pair $f \bar{f}$ via the interaction (3.5), and the corresponding decay length is

$$
l_{X \rightarrow f \bar{f}} \approx\left(\frac{1}{Q_{f}}\right)^{2}\left(\frac{10^{-6}}{\theta}\right)^{2}\left(\frac{0.4 \mathrm{GeV}}{m_{X}}\right) \times 25 \mathrm{~cm} .
$$

As a result, we will see a signal of energetic lepton pairs plus missing energy with a displacement vertex of $\mathcal{O}(10) \mathrm{cm}$ as $\widetilde{X}$ can either be the LSP in the hidden sector or decay to a hidden sector higgs and higgino.

Of course, this conclusion is valid only if the conventional decay channel of the OLSP to the gravitino $\psi_{3 / 2}$ is not too much more efficient than the above process. In GMSB models, supersymetry breaking is driven by a hidden strong dynamics which is supposed to generate supersymmetry breaking F-term with $\sqrt{F} \gtrsim 100 \mathrm{TeV}$ [47]. Then the OLSP decay length for the process $\chi_{1}^{0} \rightarrow \gamma \psi_{3 / 2}$ is

$$
l_{\chi_{1}^{0} \rightarrow \gamma \psi_{3 / 2}} \approx\left(\frac{\sqrt{F}}{10^{5} \mathrm{GeV}}\right)^{4}\left(\frac{200 \mathrm{GeV}}{m_{\chi_{1}^{0}}}\right)^{5} \times 3 \times 10^{-4} \mathrm{~cm} .
$$


Remarkably, this number is comparable to (5.5), and there may be a chance to confirm both the GMSB mechanism and the presence of sub-GeV hidden $\mathrm{U}(1)$ by observing both signals for (5.5) and (5.7).

\section{Conclusion}

The presence of $\mathrm{TeV}$ dark matter associated with a hidden $U(1)_{X}$ gauge symmetry broken below the GeV scale has been motivated by the recent results from PAMELA and ATIC. Elaborating this idea in the framework of gauge mediated supersymmetry breaking, we obtain some interesting constraints and prospects of the scenario.

Explaining the heaviness of dark matter in relation to the resolution of the $\mu$ and $B \mu$ problem of GMSB models, it follows that the vector-like scalar particle charged under $U(1)_{X}$ becomes a good dark matter candidate for the gauge coupling $\alpha_{X} \sim \alpha$ and the dark matter mass $\sim$ TeV. The other 'hidden' particles, namely the $U(1)_{X}$ gauge boson and gaugino, higgs boson and higgsino, obtain masses below the GeV scale through a radiative mechanism with properly chosen particle contents and Yukawa couplings among them. The hidden sector communicates with the visible (Standard Model) sector either by the Yukwa terms generating the Higgsino and dark matter masses or by the kinetic mixing between $U(1)_{X}$ and $U(1)_{Y}$. It turns out that the kinetic mixing parameter $\theta$ receives a strong upper limit $\theta \lesssim 10^{-6}\left(m_{X} / 0.4 \mathrm{GeV}\right)^{2}$ coming from the experimental data on direct detection of dark matter.

In this scenario, positive signals for direct dark matter detection in future experiments may arise either from the vector interaction controlled by $\theta$ or from the scalar interaction exchanging the Higgs boson. Another interesting consequence for the LHC experiment is that the ordinary LSP decay leaves quite distinctive signals of missing energy and energetic lepton pairs with invariant mass below $\mathrm{GeV}$ and decay gaps larger than about $10 \mathrm{~cm}$. Furthermore, the rates for this decay and the usual decay to photon and gravitino, a characteristic of GMSB models, are found to be comparable in typical parameter range of the model. Thus, the scenario of the sub-GeV $U(1)_{X}$ sector in GMSB models may be tested at the LHC by observing both signatures of lepton pairs plus missing energy and photons plus missing energy.

However, we find that the boost factor for the galactic dark matter annihilation required by the PAMELA and ATIC data can not be obtained solely by the Sommerfeld effect in our scenario. Typical Sommerfeld enhancement factor is in the range of $40-60$ for the dark matter masses of $600-1000 \mathrm{GeV}$. Thus, we may have to invoke additional sources of the boost factor like the clumpy distribution of dark matter.

\section{References}

[1] O. Adriani et al., arXiv:0810.4995 [astro-ph]; O. Adriani et al., arXiv:0810.4994 [astro-ph]. 
[2] I. V. Moskalenko and A. W. Strong, Astrophys. J. 493, 694 (1998) [arXiv:astro-ph/9710124].

[3] S. W. Barwick et al. [HEAT Collaboration], Astrophys. J. 482, L191 (1997) [arXiv:astro-ph/9703192]; J. J. Beatty et al., Phys. Rev. Lett. 93 (2004) 241102 [arXiv:astro-ph/0412230].

[4] M. Aguilar et al. [AMS-01 Collaboration], Phys. Lett. B 646, 145 (2007) [arXiv:astro-ph/0703154].

[5] S. Torii et al., arXiv:0809.0760 [astro-ph].

[6] J. Chang et al. [ATIC collaboration], Nature 456, 362 (2008).

[7] D. Hooper, P. Blasi and P. D. Serpico, arXiv:0810.1527 [astro-ph].

[8] H. Yuksel, M. D. Kistler and T. Stanev, arXiv:0810.2784 [astro-ph].

[9] J. Hall and D. Hooper, arXiv:0811.3362 [astro-ph].

[10] J. Lavalle, Q. Yuan, D. Maurin and X. J. Bi, arXiv:0709.3634 [astro-ph].

[11] F. Donato, D. Maurin, P. Brun, T. Delahaye and P. Salati, arXiv:0810.5292 [astro-ph].

[12] P. Grajek, G. Kane, D. J. Phalen, A. Pierce and S. Watson, arXiv:0807.1508 [hep-ph].

[13] L. Bergstrom, T. Bringmann and J. Edsjo, arXiv:0808.3725 [astro-ph].

[14] M. Cirelli and A. Strumia, arXiv:0808.3867 [astro-ph].

[15] V. Barger, W. Y. Keung, D. Marfatia and G. Shaughnessy, arXiv:0809.0162 [hep-ph].

[16] C. R. Chen, F. Takahashi and T. T. Yanagida, arXiv:0809.0792 [hep-ph].

[17] I. Cholis, L. Goodenough, D. Hooper, M. Simet and N. Weiner, arXiv:0809.1683 [hep-ph].

[18] M. Cirelli, M. Kadastik, M. Raidal and A. Strumia, arXiv:0809.2409 [hep-ph].

[19] J. H. Huh, J. E. Kim and B. Kyae, arXiv:0809.2601 [hep-ph].

[20] N. Arkani-Hamed, D. P. Finkbeiner, T. Slatyer and N. Weiner, arXiv:0810.0713 [hep-ph].

[21] M. Pospelov and A. Ritz, arXiv:0810.1502 [hep-ph].

[22] M. Fairbairn and J. Zupan, arXiv:0810.4147 [hep-ph].

[23] A. E. Nelson and C. Spitzer, arXiv:0810.5167 [hep-ph]. 
[24] I. Cholis, D. P. Finkbeiner, L. Goodenough and N. Weiner, arXiv:0810.5344 [astro-ph].

[25] Y. Nomura and J. Thaler, arXiv:0810.5397 [hep-ph].

[26] R. Harnik and G. D. Kribs, arXiv:0810.5557 [hep-ph].

[27] D. Feldman, Z. Liu and P. Nath, arXiv:0810.5762 [hep-ph].

[28] P. f. Yin, Q. Yuan, J. Liu, J. Zhang, X. j. Bi and S. h. Zhu, arXiv:0811.0176 [hep-ph].

[29] K. Ishiwata, S. Matsumoto and T. Moroi, arXiv:0811.0250 [hep-ph].

[30] Y. Bai and Z. Han, arXiv:0811.0387 [hep-ph].

[31] P. J. Fox and E. Poppitz, arXiv:0811.0399 [hep-ph].

[32] C. R. Chen, F. Takahashi and T. T. Yanagida, arXiv:0811.0477 [hep-ph].

[33] A. Ibarra and D. Tran, arXiv:0811.1555 [hep-ph].

[34] S. Baek and P. Ko, arXiv:0811.1646 [hep-ph].

[35] I. Cholis, G. Dobler, D. P. Finkbeiner, L. Goodenough and N. Weiner, arXiv:0811.3641 [astro-ph].

[36] E. Nardi, F. Sannino and A. Strumia, arXiv:0811.4153 [hep-ph].

[37] K. M. Zurek, arXiv:0811.4429 [hep-ph].

[38] M. Pospelov, A. Ritz and M. B. Voloshin, Phys. Lett. B 662, 53 (2008) [arXiv:0711.4866 [hep-ph]].

[39] N. Arkani-Hamed and N. Weiner, arXiv:0810.0714 [hep-ph].

[40] H. Baer, K. m. Cheung and J. F. Gunion, Phys. Rev. D 59, 075002 (1999) [arXiv:hep-ph/9806361].

[41] J. Hisano, S. Matsumoto and M. M. Nojiri, Phys. Rev. D 67, 075014 (2003) [arXiv:hep-ph/0212022]; J. Hisano, S. Matsumoto and M. M. Nojiri, Phys. Rev. Lett. 92, 031303 (2004) [arXiv:hep-ph/0307216]; J. Hisano, S. Matsumoto, M. M. Nojiri and O. Saito, Phys. Rev. D 71, 015007 (2005) [arXiv:hep-ph/0407168]; J. Hisano, S. Matsumoto, M. M. Nojiri and O. Saito, Phys. Rev. D 71, 063528 (2005) [arXiv:hep-ph/0412403]; J. Hisano, S. Matsumoto, O. Saito and M. Senami, Phys. Rev. D 73, 055004 (2006) [arXiv:hep-ph/0511118]; J. Hisano, S. Matsumoto, M. Nagai, O. Saito and M. Senami, Phys. Lett. B 646, 34 (2007) [arXiv:hep-ph/0610249].

[42] S. Profumo, Phys. Rev. D 72, 103521 (2005) [arXiv:astro-ph/0508628].

[43] M. Cirelli, A. Strumia and M. Tamburini, Nucl. Phys. B 787, 152 (2007) [arXiv:0706.4071 [hep-ph]]. 
[44] J. March-Russell, S. M. West, D. Cumberbatch and D. Hooper, JHEP 0807, 058 (2008) [arXiv:0801.3440 [hep-ph]].

[45] L. B. Okun, Sov. Phys. JETP 56, 502 (1982) [Zh. Eksp. Teor. Fiz. 83, 892 (1982)];

B. Holdom, Phys. Lett. B 166, 196 (1986).

[46] J. H. Huh, J. E. Kim, J. C. Park and S. C. Park, Phys. Rev. D 77, 123503 (2008) [arXiv:0711.3528 [astro-ph]].

[47] M. Dine and A. E. Nelson, Phys. Rev. D 48, 1277 (1993) [arXiv:hep-ph/9303230];

M. Dine, A. E. Nelson and Y. Shirman, Phys. Rev. D 51, 1362 (1995) [arXiv:hep-ph/9408384]; M. Dine, A. E. Nelson, Y. Nir and Y. Shirman, Phys. Rev. D 53, 2658 (1996) [arXiv:hep-ph/9507378].

[48] K. Agashe and M. Graesser, Nucl. Phys. B 507, 3 (1997) [arXiv:hep-ph/9704206];

A. de Gouvea, A. Friedland and H. Murayama, Phys. Rev. D 57, 5676 (1998) [arXiv:hep-ph/9711264].

[49] D. Feldman, Z. Liu and P. Nath, Phys. Rev. D 75, 115001 (2007) [arXiv:hep-ph/0702123].

[50] A. Ibarra, A. Ringwald and C. Weniger, arXiv:0809.3196 [hep-ph].

[51] G. Bertone, D. Hooper and J. Silk, Phys. Rept. 405, 279 (2005) [arXiv:hep-ph/0404175].

[52] E. Komatsu et al. [WMAP Collaboration], arXiv:0803.0547 [astro-ph].

[53] G. Jungman, M. Kamionkowski and K. Griest, Phys. Rept. 267, 195 (1996) [arXiv:hep-ph/9506380].

[54] C. P. Burgess, M. Pospelov and T. ter Veldhuis, Nucl. Phys. B 619, 709 (2001) [arXiv:hep-ph/0011335].

[55] D. P. Finkbeiner, T. Slatyer and N. Weiner, Phys. Rev. D 78, 116006 (2008) [arXiv:0810.0722 [hep-ph]].

[56] H. S. Lee. et al. [KIMS Collaboration], Phys. Rev. Lett. 99, 091301 (2007) [arXiv:0704.0423 [astro-ph]].

[57] Z. Ahmed et al. [CDMS Collaboration], arXiv:0802.3530 [astro-ph].

[58] D. Tucker-Smith and N. Weiner, Phys. Rev. D 64, 043502 (2001) [arXiv:hep-ph/0101138].

[59] P. Fayet, Phys. Rev. D 75, 115017 (2007) [arXiv:hep-ph/0702176]. 